\title{
Caracterização física, físico-química e química de frutos de genótipos de cajazeiras ${ }^{(1)}$
}

\author{
Wilza da Silveira Pinto( ${ }^{(2)}$, Ana Cristina Vello Loyola Dantas ${ }^{(3)}$, Antonio Augusto Oliveira Fonseca( ${ }^{(3)}$, \\ Carlos Alberto da Silva Ledo ${ }^{(4)}$, Sandra Cerqueira de Jesus ${ }^{(5)}$, Perinto Luis Pimentel Calafange ${ }^{(4)}$ \\ e Elton Monteiro Andrade(3)
}

\begin{abstract}
Resumo - Este trabalho teve como objetivo caracterizar frutos de genótipos de cajazeira (Spondias mombin L.) visando identificar materiais de interesse industrial e para trabalhos de melhoramento. Frutos de 30 genótipos foram caracterizados avaliando-se: $\mathrm{pH}$, sólidos solúveis totais (SST), acidez total titulável (ATT), vitamina C, relação sólidos solúveis total/acidez total titulável, açúcares totais, rendimento industrial, massa total do fruto, massa da semente, massa da casca, massa da polpa e porcentual de rendimento de polpa. Os resultados (médias de três amostras) foram avaliados por estatística descritiva utilizando-se medida de tendência central (média) e de variabilidade de dados (desvio-padrão e coeficiente de variação). Foram realizadas análises estatísticas multivariadas, utilizando-se as técnicas de análise de agrupamento e análise de componentes principais. Os frutos que apresentaram melhores características para o processamento foram os provenientes dos genótipos AJ04UB, VS07UB, TF25TN, TF26TN, TF29TN, TF30TN e TF31TN. A análise de agrupamento mostrou a formação de quatro grupos de genótipos, o que demonstra a variabilidade genética existente na espécie.
\end{abstract}

Termos para indexação: Spondias mombin, característica agronômica, variação genética, melhoramento de plantas.

\section{Physical, physico-chemical and chemical characterization of fruits of true yellow mombin}

\begin{abstract}
The objective of this work was to characterize fruits of true yellow mombin (Spondias mombin L.) genotypes to identify materials of industrial interest and for breeding works. Fruits of 30 genotypes were characterized by evaluation of $\mathrm{pH}$, total soluble solid (TSS), titratable total acidity (TTA), vitamin C, TSS/TTA rate, total sugars, industrial income, total fruit mass, seed mass, rind mass, pulp mass, and percentage of pulp income. The results (average of three samples) were evaluated by descriptive statistics using central trend (average) and variability of data (standard error and coefficient of variation). Multivaried statistical analyses were carried out, by means of grouping and main components techniques. Fruits from the genotypes AJ04UB, VS07UB, TF25TN, TF26TN, TF29TN, TF30TN and TF31TN presented the best features for processing. Grouping analysis showed formation of four genotypes groups showing the existence of genetic variability in the species.
\end{abstract}

Index terms: Spondias mombin, plant characters, genetic variation, plant breeding.

(1) Aceito para publicação em 30 de julho de 2003.

Extraído da dissertação de mestrado apresentada pelo primeiro autor à Universidade Federal da Bahia (UFBA), Escola de Agronomia, Cruz das Almas, BA.

(2) Universidade Federal Rural da Amazônia, Caixa Postal 907, CEP 66077-530 Belém, Pará. E-mail: wilzapinto@bol.com.br

(3) UFBA, Escola de Agronomia, Caixa Postal 82, CEP 44380-000 Cruz das Almas, BA. E-mail: acvld@ufba.br, bbui@zipmail.com.br

(4) Embrapa-Centro Nacional de Pesquisa de Mandioca e Fruticultura, Caixa Postal 07, CEP 44380-000 Cruz das Almas, BA. E-mail: ledo@cnpmf.embrapa.br, peri@cnpmf.embrapa.br

(5) Rua N. Sra. da Conceição, Tancredo Neves, 251, CEP 41210-110 Salvador, BA. E-mail: scjsandra@bol.com.br

\section{Introdução}

A cajazeira (Spondias mombin L.), planta da família das Anacardiáceas (Lorenzi, 1992), tem como centro de origem a América Tropical e encontra-se amplamente disseminada no Brasil. Na Amazônia, é vulgarmente conhecida por taperebá; em São Paulo e Minas Gerais, por cajazeira-miúda e cajá-pequeno; nos estados do Sul, por cajazeira ou cajá-mirim, e na maioria dos estados do Nordeste, por cajá. No Nordeste ocorre espontaneamente em condições silvestres, competindo com outras espécies vegetais e em quintais, 
sítios e, até mesmo, na proteção e sombreamento do cacaueiro (Bosco et al., 2000).

É um fruto tropical com crescente valor de mercado, principalmente no Nordeste brasileiro, onde ocupa lugar de destaque na produção e comercialização de polpa, pela excelente qualidade sensorial que apresenta. $\mathrm{O}$ aumento da demanda vem despertando o interesse pelo seu cultivo, porém o incipiente acervo de informações e conhecimentos existentes impede a instalação de pomares comerciais (Souza, 1998). A expansão do cultivo da cajazeira, em escala comercial, depende do uso de material propagativo com elevado potencial produtivo e com características qualitativas desejáveis (Bosco et al., 2000).

A qualidade dos frutos é atribuída aos caracteres físicos que respondem pela aparência externa, entre os quais destacam-se o tamanho, a forma do fruto e a cor da casca. Essas características estão relacionadas ao conjunto de atributos referentes à aparência, sabor, odor, textura e valor nutritivo (Chitarra \& Chitarra, 1990).

Na produção de frutos destinados à indústria de sucos, deve-se dar ênfase a tecnologias que confiram aos frutos alto rendimento em suco, boa consistência, maior teor de açúcar e acidez elevada.

A deficiência de tecnologias de produção de fruteiras tropicais consiste no principal obstáculo à exploração comercial, tanto para o mercado interno quanto para o externo.

Este trabalho teve por objetivo caracterizar frutos de genótipos de cajazeiras provenientes dos municípios de Ubaíra, Amargosa e Tancredo Neves, no Estado da Bahia, visando identificar materiais de interesse agroindustrial e para melhoramento da espécie.

\section{Material e Métodos}

Foram coletados frutos de 30 genótipos de cajazeiras nos municípios de Ubaíra (latitude $13^{\circ} 16^{\prime} 0^{\prime \prime} \mathrm{S}$ e longitude $30^{\circ} 40^{\prime} 0$ " W; 320 m de altitude e clima subúmido), Amargosa (latitude $13^{\circ} 2^{\prime} 0^{\prime \prime} \mathrm{S}$ e longitude $39^{\circ} 36^{\prime} 0^{\prime \prime} \mathrm{W} ; 400 \mathrm{~m}$ de altitu- de e clima subúmido a seco) e Tancredo Neves (latitude $13^{\circ} 27^{\prime} 0 " \mathrm{~S}$ e longitude $39^{\circ} 25^{\prime} \mathrm{O}^{\prime \prime} \mathrm{W} ; 160 \mathrm{~m}$ de altitude e clima úmido). A coleta foi realizada em áreas de plantio de cacau, que tem a cajazeira como sombreamento, em idades que variaram entre 9 e 60 anos. Os frutos foram coletados no mesmo dia da queda, ensacados em sacos de polietileno preto e conservados em câmara fria para análise no dia seguinte. Selecionaram-se 30 frutos por planta, descartando-se aqueles com danos e uniformizando-os quanto ao estádio de maturação. Os frutos selecionados foram analisados no Laboratório de Fisiologia Vegetal da EmbrapaCentro Nacional de Pesquisa de Mandioca e Fruticultura e Laboratório de Química da Escola de Agronomia/UFBA, em Cruz das Almas, BA. Foram avaliados os seguintes aspectos físico-químicos, químicos e físicos: $\mathrm{pH}$, pelo método potenciométrico (Association of Official Agricultural Chemists, 1975); sólidos solúveis totais (SST), pelo método refratométrico (Pearson, 1973); acidez total titulável (ATT), pelo método acidimétrico (Association of Official Agricultural Chemists, 1975); vitamina C (Association of Official Agricultural Chemists, 1975); açúcares totais (AT) (Association of Official Agricultural Chemists, 1975); relação SST/ATT; rendimento industrial, calculado pelo quociente de porcentual de polpa e porcentual de SST em ${ }^{\circ}$ Brix, em laboratório; massa total do fruto, massa da semente, massa da casca, massa da polpa, obtidas por gravimetria, e o porcentual de rendimento de polpa. Os resultados foram analisados por estatística descritiva utilizando-se medidas de tendência central (média) e de variabilidade de dados (desvio-padrão e coeficiente de variação) pelo SAEG (Ribeiro Júnior, 2001).

Foram realizadas análises estatísticas multivariadas, por meio de técnicas de análise de agrupamento ou de cluster e análise de componentes principais. Como medida de dissimilaridade foi utilizada a distância euclidiana média e como método hierárquico aglomerativo, o método de Ward (1963). Com base nos cálculos, foi estabelecido o dendrograma, o qual permite verificar o grau de similaridade entre os genótipos e grupos similares, ou entre dois grupos distintos. As análises de agrupamento e por componentes principais foram realizadas utilizando-se os programas Statistica for Windows (Statsoft Inc., 2002) e Cruz (2001), respectivamente. 


\section{Resultados e Discussão}

$\mathrm{O} \mathrm{pH}$ foi a variável que apresentou a menor variação, com coeficiente de $6,45 \%$ (Tabela 1 ). A amplitude de variação foi de 2,26 (genótipo MS11UB) a 2,95 (genótipo GR22UB), com média de 2,61. Esses valores estão acima dos valores mínimos do Padrão de Identidade e Qualidade (PIQ) para polpa de cajá, que é de 2,2 (Brasil, 1999). A média observada foi superior às encontradas, em polpa congelada, por Oliveira et al. (1999) (2,57) e Bastos et al. (1999) $(2,54)$, e inferiores às apresentadas por Oliveira et al. (2000), que foi de 2,81 em polpas congeladas, no Estado da Bahia, e por Aldrigue (1988), de 2,90 em polpa in natura. Os resultados obtidos com os 30 genótipos analisados são considerados satisfatórios, segundo o padrão exigido para polpa de cajá.
O teor de SST dos frutos variou de 7,07 a 14,00 Brix, com média de $11,01^{\circ}$ Brix e um coeficiente de variação de 16,53\% (Tabela 1). Alguns genótipos (LI01UB, LI02UB, AJ05UB, IS15UB e GR20UB) apresentaram níveis abaixo do mínimo exigido $\left(9,00^{\circ}\right.$ Brix $)$ pelo PIQ para polpa de cajá (Brasil, 1999). No entanto, a média $\left(11,01^{\circ}\right.$ Brix $)$ foi superior às relatadas por Oliveira et al. (1998, 1999, 2000) e Bastos et al. (1999) 8,10³rix, $8,74^{\circ}$ Brix, $9,39^{\circ}$ Brix e $7,67^{\circ}$ Brix, respectivamente, com polpas congeladas, porém, inferior à descrita por Aldrigue (1988), $11,30^{\circ}$ Brix, com polpa in natura. Destacam-se como os genótipos mais promissores MP03UB, MS11UB, WL14UB, JC17AM, GR21UB, GR22UB, TF28TN, TF29TN e TF30TN, por apresentarem valores acima de $12^{\circ}$ Brix. Apenas $16,66 \%$ dos genótipos estão fora do padrão de identidade e qualidade de polpa de cajá do Ministério da Agricultura,

Tabela 1. Características físico-químicas, químicas e rendimento industrial de 30 genótipos de cajazeira ${ }^{(1)}$.

\begin{tabular}{|c|c|c|c|c|c|c|c|}
\hline Genótipo & $\mathrm{pH}$ & $\begin{array}{c}\text { SST } \\
\left({ }^{\circ} \text { Brix }\right)\end{array}$ & $\begin{array}{c}\text { ATT } \\
(\mathrm{mg} / 100 \mathrm{~g})\end{array}$ & $\begin{array}{c}\text { Vit. C } \\
(\mathrm{mg} / 100 \mathrm{~g})\end{array}$ & SST/ATT & $\begin{array}{c}\mathrm{AT} \\
(\% \text { de sacarose })\end{array}$ & $\begin{array}{c}\text { Rendimento } \\
(\%)\end{array}$ \\
\hline LI 01UB & 2,74 & 8,00 & 0,73 & 13,46 & 10,9 & 6,61 & 3,65 \\
\hline LI 02UB & 2,86 & 7,07 & 0,67 & 6,99 & 10,6 & 5,78 & 3,93 \\
\hline MP03UB & 2,70 & 12,53 & 1,00 & 16,72 & 12,5 & 11,53 & 6,63 \\
\hline AJ04UB & 2,47 & 9,30 & 1,05 & 12,01 & 8,9 & 7,18 & 4,55 \\
\hline AJ05UB & 2,48 & 8,07 & 1,03 & 10,69 & 7,8 & 6,28 & 3,75 \\
\hline DB06UB & 2,45 & 10,20 & 0,85 & 13,34 & 12,0 & 8,57 & 4,48 \\
\hline VS07UB & 2,75 & 11,27 & 1,20 & 19,70 & 9,4 & 9,49 & 5,89 \\
\hline VS08UB & 2,90 & 9,37 & 0,73 & 13,78 & 12,8 & 7,25 & 4,78 \\
\hline VS09UB & 2,58 & 9,93 & 0,76 & 13,08 & 13,1 & 7,82 & 2,72 \\
\hline MS10UB & 2,62 & 12,07 & 0,89 & 21,43 & 13,6 & 10,40 & 6,40 \\
\hline MS11UB & 2,26 & 12,93 & 1,53 & 23,85 & 8,5 & 11,58 & 7,76 \\
\hline MS12UB & 2,37 & 11,67 & 1,18 & 21,16 & 9,9 & 10,47 & 7,22 \\
\hline WL13UB & 2,46 & 12,00 & 1,19 & 20,57 & 10,1 & 11,07 & 6,36 \\
\hline WL14UB & 2,63 & 12,93 & 0,82 & 13,46 & 15,7 & 10,69 & 6,59 \\
\hline IS15UB & 2,74 & 8,87 & 0,95 & 14,24 & 9,3 & 6,84 & 4,58 \\
\hline JC16AM & 2,41 & 11,07 & 0,83 & 17,57 & 13,4 & 8,70 & 6,69 \\
\hline JC17AM & 2,51 & 12,87 & 1,05 & 20,75 & 12,2 & 11,32 & 6,05 \\
\hline GR18UB & 2,58 & 10,07 & 0,83 & 16,74 & 12,2 & 10,75 & 3,81 \\
\hline GR19UB & 2,71 & 11,87 & 0,89 & 18,88 & 13,4 & 10,21 & 6,13 \\
\hline GR20UB & 2,44 & 8,87 & 0,90 & 17,90 & 9,8 & 6,32 & 3,99 \\
\hline GR21UB & 2,55 & 13,00 & 0,79 & 20,76 & 16,5 & 11,16 & 6,89 \\
\hline GR22UB & 2,95 & 12,13 & 0,58 & 17,78 & 21,1 & 10,57 & 5,73 \\
\hline GR23UB & 2,54 & 9,07 & 1,08 & 12,48 & 8,4 & 7,59 & 5,09 \\
\hline TF25TN & 2,90 & 11,00 & 1,60 & 13,36 & 7,3 & 10,62 & 5,91 \\
\hline TF26TN & 2,60 & 12,00 & 1,50 & 12,01 & 8,0 & 11,14 & 6,34 \\
\hline TF27TN & 2,70 & 10,00 & 1,17 & 16,29 & 8,6 & 8,23 & 4,02 \\
\hline TF28TN & 2,54 & 14,00 & 1,41 & 17,19 & 10,0 & 12,18 & 6,90 \\
\hline TF29TN & 2,55 & 13,00 & 1,75 & 17,19 & 7,5 & 11,08 & 5,88 \\
\hline TF30TN & 2,79 & 13,00 & 1,39 & 15,00 & 9,3 & 11,30 & 6,61 \\
\hline TF31TN & 2,57 & 12,00 & 1,51 & 23,63 & 8,0 & 10,64 & 5,54 \\
\hline Média & 2,61 & 11,01 & 1,06 & 16,40 & 11,03 & 9,45 & 5,50 \\
\hline Desvio-padrão & 0,17 & 1,82 & 0,31 & 4,03 & 3,09 & 1,96 & 1,29 \\
\hline $\mathrm{CV}(\%)$ & 6,45 & 16,53 & 29,00 & 24,56 & 28,01 & 20,73 & 23,42 \\
\hline
\end{tabular}

(1)SST: sólidos solúveis totais; ATT: acidez total titulável; AT: açúcares total; UB: Ubaíra; AM: Amargosa; TN: Tancredo Neves. 
porém apresentam características físicas interessantes para trabalhos de melhoramento genético.

Os valores de ATT encontrados variaram de $0,58 \%$ a $1,75 \%$ de ácido cítrico, com média de $1,06 \%$, com coeficiente de variação de $29,00 \%$, o que mostra a grande variação ocorrida entre os genótipos avaliados. Entre estes, 17 genótipos apresentaram níveis acima do mínimo $(0,90 \%)$ exigido pelo PIQ (Brasil, 1999). O valor médio detectado está abaixo do encontrado por Silva et al. (1999) com polpa in natura $(1,43 \%)$ e por Bastos et al. (1999) e Oliveira et al. (2000) com polpa congelada $(1,12 \%$ e $1,20 \%)$, respectivamente. Pode-se considerar os genótipos com valores acima de $1,00 \%$ como os de maior interesse para a agroindústria e para trabalhos de melhoramento da espécie.

Com relação aos valores de vitamina $\mathrm{C}$, obtevese a média de $16,40 \mathrm{mg} / 100 \mathrm{~g}$, e faixa de 6,99 a 23,85 mg/100 g, o que mostra a alta variabilidade existente na espécie em relação a esta variável. O PIQ não especifica limites para esta variável quanto à polpa de cajá. Os resultados observados, com exceção do genótipo LI02UB (6,99mg/100 g), são superiores às médias apresentadas por Oliveira et al. (1999) $(10,29 \mathrm{mg} / 100 \mathrm{~g})$ e Bastos et al. (1999) $(9,10 \mathrm{mg} / 100 \mathrm{~g})$.

A relação SST/ATT é uma das melhores formas de avaliação do sabor, sendo mais representativa que a medição isolada de açúcares e de acidez. As análises realizadas mostraram alta variabilidade entre os genótipos estudados, com média geral de 11,03 e valores máximo e mínimo de 21,1 e 7,3, respectivamente (Tabela 1). Tais valores são superiores aos apresentados por Bastos et al. (1999) em análise com polpas congeladas (média de 7,14 e faixa de 15,32 a 2,63), e a média é superior à média de 6,15 relatada por Silva et al. (1999) com polpa in natura. Os genótipos MP03UB, DB06UB, VS08UB, VS09UB, MS10UB, WL14UB, JC16AM, JC17AM, GR18UB, GR19UB, GR21UB, GR22UB e TF28TN apresentaram níveis acima da média e podem ser considerados como os melhores para um trabalho de melhoramento.

Os teores de AT variaram de 5,78\% a 12,18\%, com média de 9,45\%. Apenas o genótipo TF28TN apresentou valor acima do máximo estipulado pelo PIQ(Brasil, 1999), queé de $12,00 \%$.A média observada está abaixo dos valores médios encontrados por Aldrigue (1988) (13,3\%), e acima das médias apresentadas por Silva et al. (1999) $(4,53 \%)$, por Bastos et al. (1999) $(3,54 \%)$ e por Oliveira et al. (2000) (4,93\%), em polpas congeladas de cajá comercializadas no Estado da Bahia.

Quanto ao rendimento industrial, a média encontrada foi de 5,50\%, e os genótipos que mais se destacaram foram: MS11UB, com 7,76\%; MS12UB, com 7,22\%; GR21UB, com 6,89\% e TF28TN, com 6,90\%. Na agroindústria, os frutos que apresentam os maiores índices de rendimento industrial são os mais desejáveis, por representarem maior possibilidade de concentração de sólidos solúveis.

Os dados referentes à caracterização física dos frutos estão apresentados na Tabela 2. A massa do fruto, que teve média de $12,12 \mathrm{~g}$, variou de um mínimo de $6,20 \mathrm{~g}$ em VS09UB a um máximo de 18,00 g em MS11UB. A média encontrada foi superior à relatada por Bosco et al. (2000), de $10 \mathrm{~g}$; à apresentada por Aldrigue (1988), de 9,5 g, e inferior à apresentada por Bosco et al. (1999), de 13,37 g. Segundo classificação apresentada por Bosco et al. (1999), são considerados frutos grandes os que possuem massa superior a $15 \mathrm{~g}$; médios, entre $12 \mathrm{~g}$ e $15 \mathrm{~g}$; e pequeno, inferior a $12 \mathrm{~g}$. Neste trabalho, constatou-se que $20 \%$ dos genótipos analisados apresentaram frutos grandes (MS11UB, MS12UB, WL13UB, GR23UB, TF27TN e TF30TN); $30 \%$, frutos médios, e $50 \%$, frutos pequenos. Pelos dados das análises, observa-se que esta variável não interfere no rendimento de polpa, uma vez que frutos pequenos (MP03UB e IS15UB) apresentaram rendimento maior que frutos grandes (TF27TN e TF30TN).

Quanto à massa da semente na composição do fruto, observa-se média de 4,34 g, que corresponde a um porcentual médio de $35,80 \%$ da massa do fruto. Os genótipos que apresentaram maiores massas de sementes foram TF27TN, com 7,08 g; GR18UB, com 5,60 g e TF30TN, com 5,46 g. Os resultados encontrados neste trabalho são superiores aos apresentados por Aldrigue (1988), com porcentagem média de 28,1\%; por Bosco et al. (1999), de 32,29\% e por Bosco et al. (2000), de 31,67\%. Observou-se que esta variável está relacionada com massa de fruto, uma vez que os frutos mais pesados também apresentaram maior massa de semente. 
A massa média da casca foi de $1,65 \mathrm{~g}$, que corresponde a $13,61 \%$ da composição do fruto, inferior à média dos dados de Bosco et al. (1999), de 15,94\%, e à apresentada por Aldrigue (1988), de 29,3\%.

Quanto à massa da polpa a média foi de $6,11 \mathrm{~g}$, com faixa de $1,70 \mathrm{~g}$ a 10,80 g, o que mostra a diversidade existente na espécie quanto a esta variável.

A média da característica rendimento de polpa foi de 49,96\%, resultado próximo aos apresentados por Bosco et al. (1999), de 51,76\% e Bosco et al. (2000) de $51,63 \%$ e superior ao apresentado por Aldrigue (1988), de 42,6\%. Na agroindústria, os frutos com grande potencial são os provenientes dos genótipos com rendimento acima da média, devendo ser melhorados alguns aspectos químicos para atender às exigências de mercado. Os genótipos MS12UB e JC16AM foram os que apresentaram maior rendimento, com $61,84 \%$ e $60,47 \%$, respectivamente.
A análise de agrupamento realizada permitiu a elaboração do dendrograma hierárquico, apresentado na Figura 1, em que se observa a formação de grupos de genótipos com algum grau de similaridade e a dissimilaridade entre grupos. Foram eliminados dessa análise as variáveis pH e relação SST/ATT. Observa-se a formação de quatro grupos, com o grupo I formado pelos genótipos LI01UB, DB06UB, AJ04UB, AJ05UB, GR20UB, TF27TN, TF28TN, TF29TN, TF31TN. Formando o grupo II, tem-se LI02UB, VS07UB, VS08UB, IS15UB, TF26TN, GR23UB, TF25TN, TF30TN. O grupo III foi formado pelos genótipos GR18UB e VS09UB e o grupo IV pelos genótipos MP03UB, GR19UB, WL14UB, MS10UB, WL13UB, GR21UB, JC17AM, GR22UB, MS11UB, MS12UB e JC16AM. Neste grupo ficaram agrupados 36,66\% dos genótipos. Segundo Dias (1998), duas

Tabela 2. Características físicas de frutos de 30 genótipos de cajazeira ${ }^{(1)}$.

\begin{tabular}{|c|c|c|c|c|c|}
\hline Genótipo & Massa do fruto (g) & Massa da semente $(\mathrm{g})$ & Massa da casca (g) & Massa da polpa (g) & Rendimento de polpa (\%) \\
\hline LI 01UB & 12,81 & 5,19 & 1,77 & 5,84 & 45,59 \\
\hline LI 02UB & 10,00 & 3,44 & 1,00 & 5,56 & 55,60 \\
\hline MP03UB & 8,50 & 3,00 & 1,00 & 4,50 & 52,94 \\
\hline AJ04UB & 13,35 & 4,56 & 2,26 & 6,53 & 48,91 \\
\hline AJ05UB & 14,76 & 5,08 & 2,09 & 6,86 & 46,48 \\
\hline DB06UB & 13,30 & 4,92 & 2,54 & 5,84 & 43,91 \\
\hline VS07UB & 7,65 & 2,77 & 0,88 & 4,00 & 52,29 \\
\hline VS08UB & 8,33 & 3,05 & 1,03 & 4,25 & 51,02 \\
\hline VS09UB & 6,20 & 2,72 & 1,78 & 1,70 & 27,42 \\
\hline MS10UB & 13,20 & 3,50 & 1,10 & 6,00 & 45,45 \\
\hline MS11UB & 18,00 & 5,40 & 1,80 & 10,80 & 60,00 \\
\hline MS12UB & 15,20 & 4,50 & 1,30 & 9,40 & 61,84 \\
\hline WL13UB & 15,10 & 4,80 & 2,30 & 8,00 & 52,98 \\
\hline WL14UB & 10,00 & 3,00 & 1,50 & 5,50 & 55,00 \\
\hline IS15UB & 9,07 & 3,74 & 0,65 & 4,68 & 51,60 \\
\hline JC16AM & 12,90 & 3,70 & 1,40 & 7,80 & 60,47 \\
\hline JC17AM & 11,70 & 4,30 & 1,90 & 5,50 & 47,01 \\
\hline GR18UB & 11,90 & 5,60 & 1,80 & 4,50 & 37,82 \\
\hline GR19UB & 12,00 & 4,60 & 1,20 & 6,20 & 51,67 \\
\hline GR20UB & 11,69 & 4,63 & 1,80 & 5,26 & 45,00 \\
\hline GR21UB & 13,20 & 4,40 & 1,80 & 7,00 & 53,03 \\
\hline GR22UB & 10,80 & 4,40 & 1,30 & 5,10 & 47,22 \\
\hline GR23UB & 15,48 & 4,89 & 1,91 & 8,68 & 56,07 \\
\hline TF25TN & 13,92 & 4,70 & 1,73 & 7,48 & 53,74 \\
\hline TF26TN & 8,92 & 3,07 & 1,13 & 4,71 & 52,80 \\
\hline TF27TN & 16,08 & 7,08 & 2,53 & 6,47 & 40,24 \\
\hline TF28TN & 11,74 & 3,98 & 1,96 & 5,79 & 49,32 \\
\hline TF29TN & 12,96 & 4,73 & 2,37 & 5,86 & 45,22 \\
\hline TF30TN & 15,26 & 5,46 & 2,04 & 7,76 & 50,85 \\
\hline TF31TN & 12,31 & 4,98 & 1,65 & 5,68 & 46,14 \\
\hline Média & 12,12 & 4,34 & 1,65 & 6,11 & 49,96 \\
\hline Desvio-padrão & 2,76 & 1,00 & 0,51 & 1,78 & 7,01 \\
\hline $\mathrm{CV}(\%)$ & 22,56 & 23,00 & 30,82 & 29,15 & 14,13 \\
\hline
\end{tabular}

${ }^{(1)}$ UB: Ubaíra; AM: Amargosa; TN: Tancredo Neves. 
populações são declaradas similares quando ocorrem na mesma região do espaço multidimensional, exibindo pequena distância entre si. A classificação obtida é conseqüência da similaridade baseada no conjunto de caracteres avaliados.

$\mathrm{Na}$ análise de componentes principais, os caracteres que mais contribuíram para a formação dos componentes 1 e 2, foram: massa da casca, massa da polpa, SST, açúcares totais e rendimento in- dustrial. A Tabela 3 mostra os respectivos coeficientes de ponderação associados às variáveis, assim como estimativas dos autovalores de cada componente considerado na análise.

Os métodos de análise de agrupamento e de componentes principais mostraram concordância na determinação de similaridade entre genótipos, com a formação de quatro grupos distintos (Figura 2).

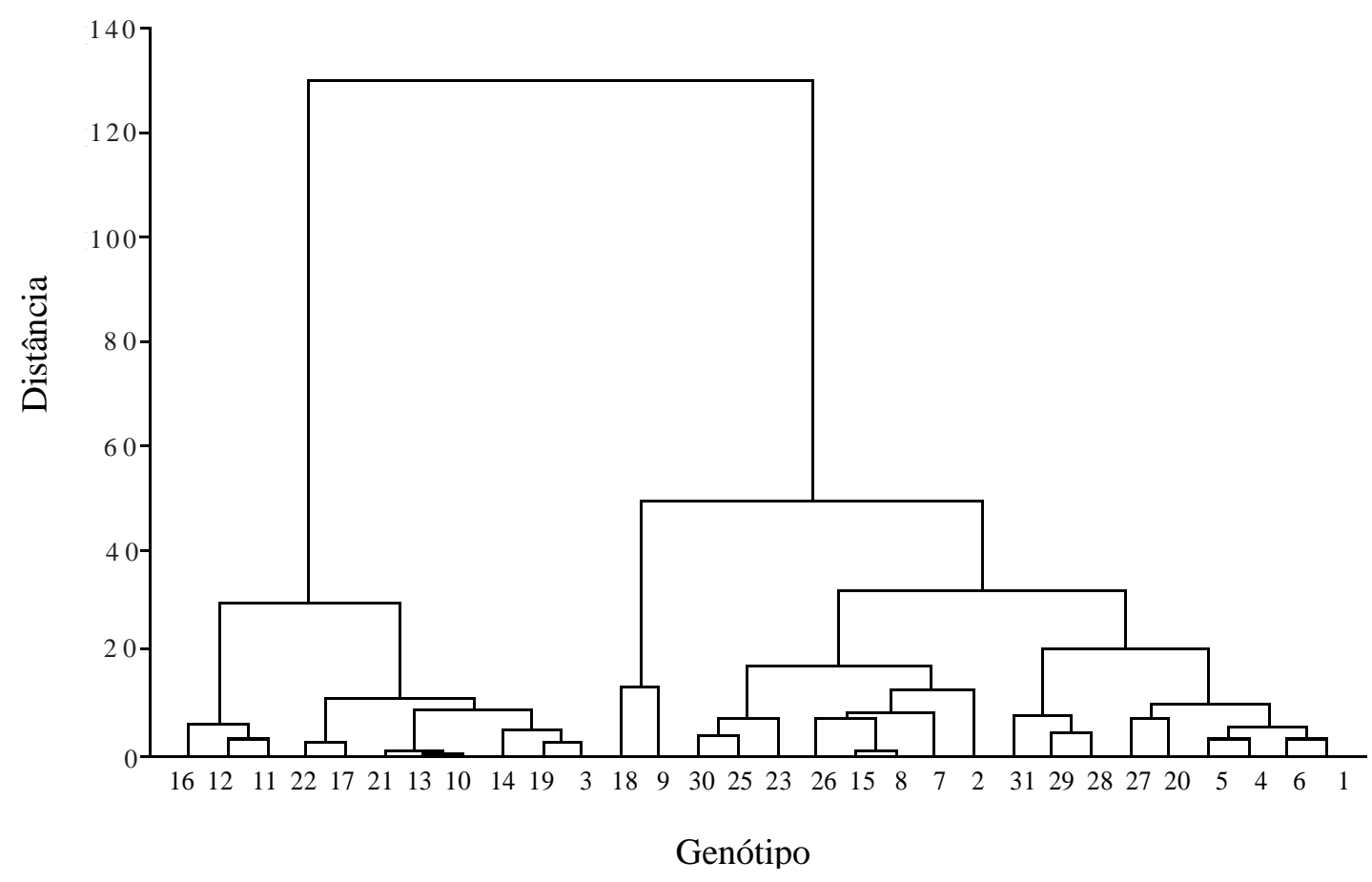

Figura 1. Dendrograma da similaridade entre 30 genótipos de cajazeira, obtido pelo método de Ward (1963), baseado na distância euclidiana média. Genótipo 1: LI01UB; 2: LI02UB; 3: MP03UB; 4: AJ04UB; 5: AJ05UB; 6: DB06UB; 7: VS07UB; 8: VS08UB; 9: VS09UB; 10: MS10UB; 11: MS11UB; 12: MS12UB; 13: WL13UB; 14: WL14UB; 15: IS15UB; 16: JC16AM; 17: JC17AM; 18: GR18UB; 19: GR19UB; 20: GR20UB; 21: GR21UB; 22: GR22UB; 23: GR23UB; 25: TF25TN; 26: TF26TN; 27: TF27TN; 28: TF28TN; 29: TF29TN; 30: TF30TN; 31: TF31TN.

Tabela 3. Componentes principais (CP) e estimativas dos autovalores obtidos da análise de dez caracteres e coeficientes de ponderação das variáveis que mais contribuíram para a formação destes componentes.

\begin{tabular}{|c|c|c|c|c|c|c|c|}
\hline \multirow[t]{2}{*}{ Componente principal } & \multicolumn{2}{|c|}{ Estimativa dos autovalores } & \multicolumn{5}{|c|}{ Coeficiente de ponderação } \\
\hline & Raiz (\%) & $\%$ acumulada & Massa de casca & Massa de polpa & $\begin{array}{c}\text { Sólidos solúveis } \\
\text { totais }\end{array}$ & Açúcares totais & $\begin{array}{c}\text { Rendimento } \\
\text { industrial }\end{array}$ \\
\hline $\mathrm{CP} 1$ & 5,6171 & 56,17 & 0,3664 & 0,3642 & 0,3179 & 0,3202 & 0,3382 \\
\hline $\mathrm{CP} 2$ & 2,4750 & 80,92 & 0,2645 & 0,2852 & 0,3415 & 0,3294 & 0,3100 \\
\hline
\end{tabular}




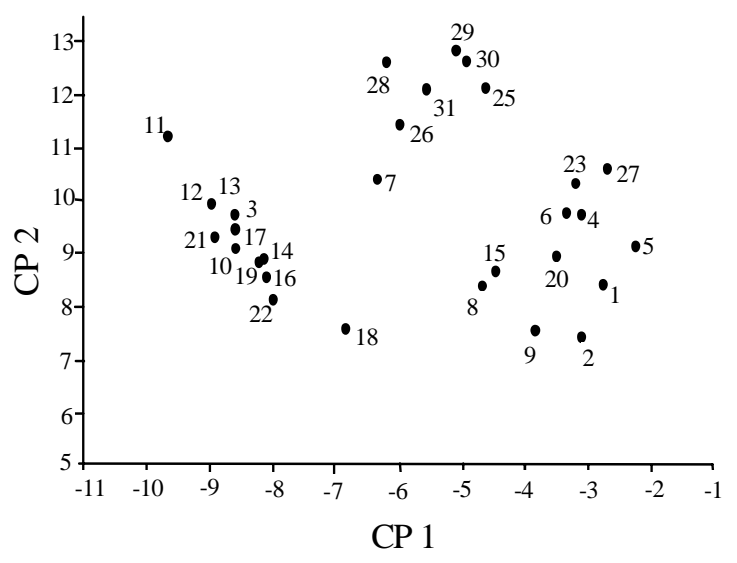

Figura 2. Dispersão gráfica dos escores de 30 genótipos de cajazeira, em relação aos componentes principais $(\mathrm{CP})$ 1 e 2. Genótipo 1: LI01UB; 2: LI02UB; 3: MP03UB; 4: AJ04UB; 5: AJ05UB; 6: DB06UB; 7: VS07UB; 8: VS08UB; 9: VS09UB; 10: MS10UB; 11: MS11UB; 12: MS12UB; 13: WL13UB; 14: WL14UB; 15: IS15UB; 16: JC16AM; 17: JC17AM; 18: GR18UB; 19: GR19UB; 20: GR20UB; 21: GR21UB; 22: GR22UB; 23: GR23UB; 25: TF25TN; 26: TF26TN; 27: TF27TN; 28: TF28TN; 29: TF29TN; 30: TF30TN; 31: TF31TN

\section{Conclusões}

1. Os frutos de cajazeira que apresentam melhores características para o processamento são os provenientes dos genótipos AJ04UB, VS07UB, TF25TN, TF26TN, TF29TN, TF30TN e TF31TN.

2. A diversidade genética existente na espécie propicia a coleta de materiais para futuros trabalhos de melhoramento e montagem de banco de germoplasma.

3. Acidez, vitamina C, SST/ATT, rendimento industrial, massa do fruto, massa da semente, massa da casca e massa da polpa são as medidas de maior variabilidade.

\section{Referências}

ALDRIGUE, M. L. Caracterização física, química e físico-química do cajá (Spondias lutea L.). In: SEMINÁRIO AGROPECUÁRIO DO ACRE, 2., 1986, Rio Branco. Anais... Brasília: Embrapa-UEPAE de Rio Branco, 1988. p. 323-327.
ASSOCIATION OF OFFICIAL AGRICULTURAL CHEMISTS (Gaithersburg, Estados Unidos). Official methods of analysis. 20th ed. Washington, 1975. 1094 p.

BASTOS, M. do S. R.; FEITOSA, T.; OLIVEIRA, M. E. B. de. Análise qualitativa e tecnológica da agroindústria de polpa de fruta na Região Nordeste. Revista Brasileira de Fruticultura, Jaboticabal, v. 21, n. 3, p. 252-257, dez. 1999.

BOSCO, J.; AGUIAR FILHO, S. P. D. de; BARROS, R. V. Banco ativo de germoplasma de cajá no Estado da Paraíba. In: WOKSHOP PARA CURADORES DE BANCO DE GERMOPLASMA DE ESPÉCIES FRUTÍFERAS, 1997, Brasília. Anais... Brasília: Embrapa-Cenargen, 1999. p. $80-85$.

BOSCO, J.; SOARES, K. T.; AGUIAR FILHO, S. P. de; BARROS, R. V. A cultura da cajazeira. João Pessoa: Emepa, 2000. 229 p. (Documentos, 28).

BRASIL. Ministério da Agricultura e do Abastecimento. Instrução Normativa no ${ }^{-122}$, de 10 de setembro de 1999. Diário Oficial [da] República Federativa do Brasil, Brasília, 13 de set. de 1999. Seção 1, p. 72-76.

Chitarra, M. I. F.; CHITARra, A. B. Pós-colheita de frutos e hortaliças: fisiologia e manuseio. Lavras: ESAL/Faepe, 1990. 320 p.

CRUZ, C. D. Programa Genes: versão Windows: aplicativo computacional em genética e estatística. Viçosa, MG: UFV, 2001. 648 p.

DIAS, L. A. dos S. Análises multidimensionais. In: ALFENAS, A. C. (Ed.). Eletroforese de isoenzimas e proteínas afins: fundamentos e aplicações em plantas e microorganismos. Viçosa, MG: UFV, 1998. p. 405-475.

LORENZI, H. Árvores brasileiras: manual de identificação e cultivo de plantas arbóreas nativas do Brasil. Nova Odessa: Plantarum, 1992. 384 p.

OLIVEIRA, M. E. B. de; BASTOS, M. do S. R.; FEITOSA, T.; BRANCO, M. A. de A. C.; SILVA, M. das G. G. da. Avaliação de parâmetros de qualidade físico-químicos de polpas congeladas de acerola, cajá e caju. Ciência e Tecnologia de Alimentos, Campinas, v. 19, n. 3, set./dez., p. 326-332, 1999.

OLIVEIRA, M. E. B. de; BASTOS, M. do S. R.; FEITOSA, T.; BRANCO, M. A. de A. C.; SILVA, M. das G. G. da. Perfil químico de qualidade das polpas de acerola, 
cajá e caju comercializadas no Estado da Bahia. Revista Brasileira de Fruticultura, Jaboticabal, v. 22, p. 9-15, jul. 2000. Número especial.

OLIVEIRA, M. E. B. de; OLIVEIRA, D. de; FEITOSA, T.; BASTOS, M. do S. R.; FREITAS, M. L. de. Avaliação química da qualidade de polpas de frutas congeladas, fabricadas e comercializadas nos Estados do Ceará e Rio Grande do Norte. Boletim do CEPPA, Curitiba, v. 16, n. 1, p. 13-21, jan./jul. 1998.

PEARSON, D. Laboratory techniques in food analysis. London: Butterworths, 1973. p. 58-60.

RIBEIRO JÚNIOR, J. I. Análises estatísticas no SAEG. Viçosa, MG: UFV, 2001. 301 p.
SILVA, A. de P. V. da; MAIA, G. A.; OLIVEIRA, G. S. F de; FIGUEIREDO, R. W. de; BRASIL, I. M. Estudo da produção do suco clarificado de cajá (Spondias lutea L.). Ciência e Tecnologia de Alimentos, Campinas, v. 19, n. 1, p. 326-332, jan./abr. 1999.

SOUZA, F. X. de. Enxertia de cajazeira (Spondias mombin L.) sobre porta-enxertos de umbuzeiro (Spondias tuberosa Arr. Câm.). Agrotrópica, Ilhéus, v. 10, n. 3, p. 189-192, set./dez. 1998

STATSOFT INC. (Tulsa, Estados Unidos). Statistica for Windows: version 2.0. Tulsa, 2002. 2 CD-ROM.

WARD, J. H. Hierarchical grouping to optimize an objective function. Journal of the American Statistical Association, Alexandria, v. 58, p. 236-244, 1963. 